\title{
GRAPHS WHOSE MINIMAL RANK IS TWO*
}

\author{
WAYNE BARRETT ${ }^{\dagger}$, HEIN VAN DER HOLST ${ }^{\ddagger}$, AND RAPHAEL LOEWY ${ }^{\S}$
}

\begin{abstract}
Let $F$ be a field, $G=(V, E)$ be an undirected graph on $n$ vertices, and let $S(F, G)$ be the set of all symmetric $n \times n$ matrices whose nonzero off-diagonal entries occur in exactly the positions corresponding to the edges of $G$. For example, if $G$ is a path, $S(F, G)$ consists of the symmetric irreducible tridiagonal matrices. Let $\operatorname{mr}(F, G)$ be the minimum rank over all matrices in $S(F, G)$. Then $\operatorname{mr}(F, G)=1$ if and only if $G$ is the union of a clique with at least 2 vertices and an independent set. If $F$ is an infinite field such that $\operatorname{char} F \neq 2$, then $\operatorname{mr}(F, G) \leq 2$ if and only if the complement of $G$ is the join of a clique and a graph that is the union of at most two cliques and any number of complete bipartite graphs. A similar result is obtained in the case that $F$ is an infinite field with char $F=2$. Furthermore, in each case, such graphs are characterized as those for which 6 specific graphs do not occur as induced subgraphs. The number of forbidden subgraphs is reduced to 4 if the graph is connected. Finally, similar criteria is obtained for the minimum rank of a Hermitian matrix to be less than or equal to two. The complement is the join of a clique and a graph that is the union of any number of cliques and any number of complete bipartite graphs. The number of forbidden subgraphs is now 5 , or in the connected case, 3 .
\end{abstract}

Key words. Rank 2, Minimum rank, Symmetric matrix, Forbidden subgraph, Bilinear symmetric form.

AMS subject classifications. 05C50, 05C75, 15A03, 15A57.

1. Introduction. Given any field $F$, let char $F$ be the characteristic of $F$, let $F^{*}$ be the nonzero elements of $F$, and let $F^{n}=\left\{\left[x_{1}, \ldots, x_{n}\right]^{t} \mid x_{1}, \ldots, x_{n} \in F\right\}$. Given a (simple, undirected) graph $G=(V, E)$ with vertex set $V=\{1,2, \ldots, n\}$, let $S(F, G)$ be the set of all symmetric $n \times n$ matrices $A=\left[a_{i j}\right]$ with entries in $F$ such that $a_{i j} \neq 0, i \neq j$, if and only if $i j \in E$. There is no restriction on the diagonal entries of $A$. We study the problem of minimizing the rank for all $A \in S(F, G)$. Let

$$
\operatorname{mr}(F, G)=\min \{\operatorname{rank} A \mid A \in S(F, G)\} .
$$

If $F=\mathbb{R}$, we abbreviate $\operatorname{mr}(F, G)$ and $S(F, G)$ to $\operatorname{mr}(G)$ and $S(G)$, respectively. Then minimizing the rank is equivalent to maximizing the multiplicity of an eigenvalue of $A \in S(G)$. It is easy to see that the maximum multiplicity of an eigenvalue of $A \in S(G)$ is $n-\operatorname{mr}(G)$. This problem is completely solved for trees by Duarte and Johnson [8]. Several additional results have been obtained for the multiplicities of eigenvalues of a matrix in $S(G)$ in the case $G$ is a tree ([10], [9], [11]). Results in a different direction were obtained by Colin de Verdière, Lovász, Schrijver, and van der

* Received by the editors 24 September 2004. Accepted for publication 8 October 2004. Handling Editor: Richard Brualdi.

${ }^{\dagger}$ Department of Mathematics, Brigham Young University, Provo, UT 84602, USA (wayne@math.byu.edu).

$\ddagger$ Faculty of Mathematics and Computer Science, Eindhoven University of Technology, 5600 MB Eindhoven, The Netherlands (Hein.van.der.Holst@cwi.nl).

$\S$ Department of Mathematics, Technion-Israel Institute of Technology, Haifa 32000, Israel (loewy@techunix.technion.ac.il). Supported by the Fund for the Promotion of Research at the Technion. Part of the research of this author was done while visiting Brigham Young University. 
Holst $([2,12,13,5])$. They are associated with a graph parameter $\mu(G)$ introduced in [2] and defined in terms of the multiplicity of the second smallest eigenvalue of matrices in $S(G)$ with a particular sign pattern and satisfying a certain transversality condition. Many of these results are gathered together in the survey paper [7]. Colin de Verdière introduced another graph parameter $\nu(G)$ in [3] more in line with this paper. It is the largest nullity attained by a positive semidefinite matrix in $S(G)$ that also satisfies a transversality condition. Van der Holst considered the parameter $\tau(G)$ in [6], the largest nullity of an $n$-by- $n$ Hermitian positive semidefinite matrix with graph $G$ (without the transversality condition), and building upon the results in [3] was able to characterize those graphs $G$ with $\tau(G) \leq 1$ and $\tau(G) \leq 2$. Later, we will also consider special cases of our results when $F=\mathbb{R}$. We let $S_{+}(G)$ be the set of positive semidefinite matrices in $S(G)$ and let

$$
\operatorname{mr}_{+}(G)=\min \left\{\operatorname{rank} A \mid A \in S_{+}(G)\right\} .
$$

In this paper we identify all graphs in $S(F, G)$ for which $\operatorname{mr}(F, G) \leq 2$ for any infinite field $F$ (or, equivalently, the nullity is greater than or equal to $n-2$ ). We first show that the complements of such graphs have a simple explicit form (Theorems 1 and 2). We also give forbidden subgraph characterizations of this class of graphs in Theorems 6 and 7 and in Theorems 9 and 10 (the connected case). In the final section of the paper we depart from the theme of symmetric matrices and determine similar criteria for a Hermitian matrix to have minimum rank at most 2.

Before proceeding, we introduce some notation from graph theory.

Definition Given a graph $G=(V, E)$, the complement of $G$ is the graph $G^{c}=$ $\left(V, E^{c}\right)$. Given two graphs $G=\left(V_{1}, E_{1}\right)$ and $H=\left(V_{2}, E_{2}\right)$, with $V_{1}$ and $V_{2}$ disjoint, the union of $G$ and $H$ is $G \cup H=\left(V_{1} \cup V_{2}, E_{1} \cup E_{2}\right)$. The join, $G \vee H$, is the graph obtained from $G \cup H$ by adding an edge from each vertex of $G$ to each vertex of $H$. If $S \subset V, G[S]$ denotes the subgraph of $G$ induced by $S$. A vertex $u \in V$ is a dominating vertex, if $u$ is adjacent to all other vertices in $V$.

Definition We denote the path on $n$ vertices by $P_{n}$. The complete graph on $n$ vertices will be denoted by $K_{n}$ and we will refer to $K_{3}$ as the triangle. We abbreviate $K_{n} \cup \cdots \cup K_{n}$ ( $m$ times) to $m K_{n}$. The complete multipartite graph, $K_{m_{1}, m_{2}, \ldots, m_{s}}$ is the complement of $K_{m_{1}} \cup K_{m_{2}} \cup \cdots \cup K_{m_{s}}$. We will be particularly interested in the complete bipartite graphs $K_{m, n}$ as well as the complete tripartite graph $K_{3,3,3}$. In $K_{m, n}$ we may allow $m$ or $n$ to equal 0 , in which case $K_{m, 0}=m K_{1}$ or $K_{0, n}=n K_{1}$.

Definition A clique of a graph $G$ is a set of pairwise adjacent vertices. A maximal clique is a clique that is not a proper subset of another clique. Given positive integers $m, n, t$ with $t<\min \{m, n\}$, we will call the graph on $m+n-t$ vertices with exactly two maximal cliques, $\{1,2, \ldots, m\},\{m-t+1, m-t+2, \ldots, m-t+n\}$, the clique sum of $K_{m}$ and $K_{n}$ on $K_{t}$. We will also refer to such graphs as clique sums. 
Note that if $\operatorname{mr}(F, G)=1$, then the rank 1 matrix $A$ attaining this rank must have the form $c x x^{t}, c \in F^{*}, x \in F^{n}$. Let $W=\left\{i \in V(G) \mid x_{i} \neq 0\right\}$ and $m=|W|$. It follows that $G=K_{m} \cup K_{n-m}^{c}$. Moreover, if $m \geq 2$, any $A \in S\left(F, K_{m} \cup K_{n-m}^{c}\right)$ has a nonzero entry and $J_{m} \oplus O_{n-n}$ (with $J_{m}$ the $m$-by- $m$ all ones matrix) is a matrix in $S\left(F, K_{m} \cup K_{n-m}^{c}\right)$ with rank 1. Thus, we have

Observation $1 \operatorname{mr}(F, G)=1$ if and only if $G=K_{m} \cup K_{n-m}^{c}, m \geq 2$.

In particular, if $G$ is connected, $\operatorname{mr}(F, G)=1$ if and only if $G$ is complete.

We note that one can reduce the problem of finding $\operatorname{mr}(F, G)$ to the connected case because it follows immediately from the definition that

Observation 2 If $G=\cup_{i=1}^{k} G_{i}$, then $\operatorname{mr}(F, G)=\sum_{i=1}^{k} \operatorname{mr}\left(F, G_{i}\right)$.

We now address the problem of finding all graphs for which $\operatorname{mr}(F, G)=2$. We begin with some sufficient conditions.

Let $J_{m, n}$ be the $m$-by-n all ones matrix and let $J_{n}=J_{n, n}$. Let $F_{2}$ be the field with two elements.

Observation 3 If $m, n \geq 1, m+n \geq 3$, then $\operatorname{mr}\left(F, K_{m, n}\right)=2$.

Apply Observation 1, and the fact that the matrix $\left[\begin{array}{cc}0 & J_{m, n} \\ J_{n, m} & 0\end{array}\right] \in S\left(F, K_{m, n}\right)$ and has rank 2.

Observation 4 Let $G$ be the clique sum of $K_{m}$ and $K_{n}$ on $K_{t}$.

(a) If $F \neq F_{2}$, then $\operatorname{mr}(F, G)=2$,

(b) If $t=1$, then $\operatorname{mr}(F, G)=2$,

(c) If $m=n=t+1$, then $\operatorname{mr}(F, G)=2$,

(d) If $t \geq 2$, and $\max \{m, n\} \geq t+2$, then $\operatorname{mr}\left(F_{2}, G\right)=3$.

For (a) let $\alpha$ be distinct from 0 and -1 . Then $\left[\begin{array}{cc}J_{m} & 0 \\ 0 & 0\end{array}\right]+\alpha\left[\begin{array}{cc}0 & 0 \\ 0 & J_{n}\end{array}\right] \in S(F, G)$ and has rank 2. (If char $F \neq 2$, just take $\alpha=1$.)

For (b) use the fact that $\left[\begin{array}{cc}J_{m} & 0 \\ 0 & 0\end{array}\right]+\left[\begin{array}{cc}0 & 0 \\ 0 & J_{n}\end{array}\right] \in S(F, G)$ and has rank 2 .

For (c) use the fact that $\left[\begin{array}{ccc}0 & J_{1, t} & 0 \\ J_{t, 1} & J_{t} & J_{t, 1} \\ 0 & J_{1, t} & 0\end{array}\right] \in S(F, G)$ and has rank 2 .

We will make no use of $(\mathrm{d})$, so only give an abbreviated argument. The matrix $K=\left[\begin{array}{ccc}J_{m-t} & J_{m-t, t} & 0 \\ J_{t, m-t} & J_{t} & J_{t, n-t} \\ 0 & J_{n-t, t} & J_{n-t}\end{array}\right] \in S\left(F_{2}, G\right)$ and has rank $3\left(\left[\begin{array}{ccc}1 & 1 & 0 \\ 1 & 1 & 1 \\ 0 & 1 & 1\end{array}\right]\right.$

is a submatrix), so $\operatorname{mr}\left(F_{2}, G\right) \leq 3$. 
Now suppose that $M=\left[\begin{array}{ccc}A & J_{m-t, t} & 0 \\ J_{t, m-t} & B & J_{t, n-t} \\ 0 & J_{n-t, t} & C\end{array}\right] \in S\left(F_{2}, G\right)$; so each nondiagonal entry of $A, B, C$ is 1 . Without loss of generality, say $n \geq t+2$. If some diagonal entry of $C$ is 0 , either $\left[\begin{array}{lll}1 & 0 & 0 \\ 1 & 1 & 1 \\ 1 & 0 & 1\end{array}\right]$ or $\left[\begin{array}{lll}1 & 0 & 0 \\ 1 & 1 & 1 \\ 1 & 1 & 0\end{array}\right]$ is a submatrix of $M$ and rank $M \geq 3$. So assume that $C=J_{n-t}$. A similar argument shows that rank $M \geq 3$ if some diagonal entry of $B$ is 0 , so we assume $B=J_{t}$. Then it is obvious that if a diagonal entry of $A$ is 0 , that rank $M \geq 3$. And if $A=J_{m-t}$, then $M=K$ and rank $M \geq 3$. Consequently, $\operatorname{mr}\left(F_{2}, G\right)=3$.

It follows from Observations 4(c), 4(b) and 3 that the graphs

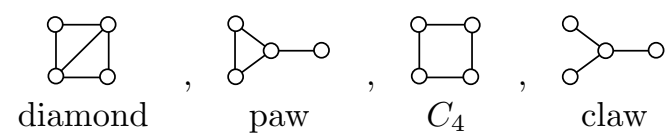

on 4 vertices all have minimum rank 2 in any field.

On the other hand, easy necessary conditions follow from

Observation 5 If $H$ is an induced subgraph of $G, \operatorname{mr}(F, G) \geq \operatorname{mr}(F, H)$.

For if $B$ is a principal submatrix of $A, \operatorname{rank} B \leq \operatorname{rank} A$.

It follows from Observation 5 that if $\operatorname{mr}(F, H)=3, H$ may not occur as an induced subgraph of any graph whose minimum rank is 2 . In other words, $H$ is a forbidden subgraph for the class of minimum rank 2 graphs. It is common to call such graphs $H$-free. Moreover, if $\mathcal{F}$ is a set of graphs, a graph is $\mathcal{F}$-free if it is $H$-free for each $H \in \mathcal{F}$.

2. Forbidden Subgraphs. We identify 6 forbidden subgraphs for the minimum rank 2 graphs.

1. $P_{4}$, which we label (1)_(2) (3) (4).

If $A \in S\left(F, P_{4}\right)$,

$$
A=\left[\begin{array}{cccc}
a_{1} & b_{1} & 0 & 0 \\
b_{1} & a_{2} & b_{2} & 0 \\
0 & b_{2} & a_{3} & b_{3} \\
0 & 0 & b_{3} & a_{4}
\end{array}\right] \quad \text { with } b_{1} b_{2} b_{3} \neq 0
$$

Then rank $A \geq \operatorname{rank}\left[\begin{array}{ccc}b_{1} & 0 & 0 \\ a_{2} & b_{2} & 0 \\ b_{2} & a_{3} & b_{3}\end{array}\right]=3$. Since

$$
\left[\begin{array}{llll}
1 & 1 & 0 & 0 \\
1 & 1 & 0 & 0 \\
0 & 0 & 0 & 0 \\
0 & 0 & 0 & 0
\end{array}\right]+\left[\begin{array}{llll}
0 & 0 & 0 & 0 \\
0 & 1 & 1 & 0 \\
0 & 1 & 1 & 0 \\
0 & 0 & 0 & 0
\end{array}\right]+\left[\begin{array}{cccc}
0 & 0 & 0 & 0 \\
0 & 0 & 0 & 0 \\
0 & 0 & 1 & 1 \\
0 & 0 & 1 & 1
\end{array}\right] \in S\left(F, P_{4}\right), \operatorname{mr}\left(F, P_{4}\right)=3
$$


We note that any proper induced subgraph of $P_{4}$ has minimum rank $\leq 2$.

2. Let $G$ be the graph

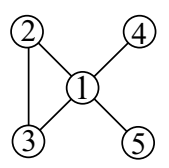

which we will denote by $\ltimes$. Then if $A \in S(F, \ltimes)$,

$$
A=\left[\begin{array}{ccccc}
d_{1} & a_{12} & a_{13} & a_{14} & a_{15} \\
a_{12} & d_{2} & a_{23} & 0 & 0 \\
a_{13} & a_{23} & d_{3} & 0 & 0 \\
a_{14} & 0 & 0 & d_{4} & 0 \\
a_{15} & 0 & 0 & 0 & d_{5}
\end{array}\right]
$$

with $a_{12} a_{13} a_{14} a_{15} a_{23} \neq 0$. Then

$$
\operatorname{rank} A \geq \operatorname{rank} A\left[\begin{array}{llllll}
1 & 2 & 4 & 1 & 3 & 5
\end{array}\right]=\operatorname{rank}\left[\begin{array}{ccc}
d_{1} & a_{13} & a_{15} \\
a_{12} & a_{23} & 0 \\
a_{14} & 0 & 0
\end{array}\right]=3 .
$$

Since

$$
\left[\begin{array}{lllll}
1 & 1 & 1 & 0 & 0 \\
1 & 1 & 1 & 0 & 0 \\
1 & 1 & 1 & 0 & 0 \\
0 & 0 & 0 & 0 & 0 \\
0 & 0 & 0 & 0 & 0
\end{array}\right]+\left[\begin{array}{lllll}
1 & 0 & 0 & 1 & 0 \\
0 & 0 & 0 & 0 & 0 \\
0 & 0 & 0 & 0 & 0 \\
1 & 0 & 0 & 1 & 0 \\
0 & 0 & 0 & 0 & 0
\end{array}\right]+\left[\begin{array}{lllll}
1 & 0 & 0 & 0 & 1 \\
0 & 0 & 0 & 0 & 0 \\
0 & 0 & 0 & 0 & 0 \\
0 & 0 & 0 & 0 & 0 \\
1 & 0 & 0 & 0 & 1
\end{array}\right] \in S(F, \ltimes)
$$

$\operatorname{mr}(F, \ltimes)=3$ for any field. Again, any proper induced subgraph of $\ltimes$ has minimum rank $\leq 2$.

3. Let $G$ be the dart

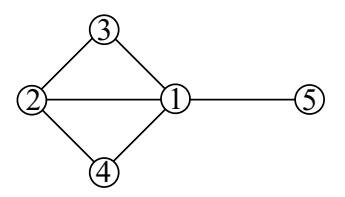

Following a similar argument to $\ltimes$, we see that $\operatorname{mr}(F$, dart $) \geq \operatorname{rank} A\left[\begin{array}{lll|llll}1 & 2 & 4 & 1 & 3 & 5\end{array}\right]$ =3. Furthermore, the matrix $\left[\begin{array}{ccccc}1+1 & 1 & 1 & 1 & 1 \\ 1 & 1 & 1 & 1 & 0 \\ 1 & 1 & 0 & 0 & 0 \\ 1 & 1 & 0 & 0 & 0 \\ 1 & 0 & 0 & 0 & 1\end{array}\right] \in S(F$, dart $)$ and has rank 3 . 
So $\operatorname{mr}(F$, dart $)=3$ for any field. Again, any proper induced subgraph of the dart has minimum rank $\leq 2$.

We also need to consider two disconnected graphs.

4. $G=P_{3} \cup K_{2}$. By Observation 2,

$$
\operatorname{mr}\left(F, P_{3} \cup K_{2}\right)=\operatorname{mr}\left(F, P_{3}\right)+\operatorname{mr}\left(F, K_{2}\right) .
$$

Clearly, we have $\operatorname{mr}\left(F, P_{3}\right)=2$ and $\operatorname{mr}\left(F, K_{2}\right)=1$, so $\operatorname{mr}\left(F, P_{3} \cup K_{2}\right)=3$.

5. $G=3 K_{2}$. Then $\operatorname{mr}\left(F, 3 K_{2}\right)=3 \operatorname{mr}\left(F, K_{2}\right)=3$.

Our final graph is

6. $G=K_{3,3,3}$. Assume that the tripartite sets are $P_{1}=\{1,2,3\}, P_{2}=\{4,5,6\}$ and $P_{3}=\{7,8,9\}$. Then any $A \in S\left(F, K_{3,3,3}\right)$ has the form

$$
A=\left[\begin{array}{ccccccccc}
d_{1} & 0 & 0 & a_{14} & a_{15} & a_{16} & a_{17} & a_{18} & a_{19} \\
0 & d_{2} & 0 & a_{24} & a_{25} & a_{26} & a_{27} & a_{28} & a_{29} \\
0 & 0 & d_{3} & a_{34} & a_{35} & a_{36} & a_{37} & a_{38} & a_{39} \\
a_{14} & a_{24} & a_{34} & d_{4} & 0 & 0 & a_{47} & a_{48} & a_{49} \\
a_{15} & a_{25} & a_{35} & 0 & d_{5} & 0 & a_{57} & a_{58} & a_{59} \\
a_{16} & a_{26} & a_{36} & 0 & 0 & d_{6} & a_{67} & a_{68} & a_{69} \\
a_{17} & a_{27} & a_{37} & a_{47} & a_{57} & a_{67} & d_{7} & 0 & 0 \\
a_{18} & a_{28} & a_{38} & a_{48} & a_{58} & a_{68} & 0 & d_{8} & 0 \\
a_{19} & a_{29} & a_{39} & a_{49} & a_{59} & a_{69} & 0 & 0 & d_{9}
\end{array}\right]
$$

with $a_{i j} \neq 0$, for all $i, j$ in distinct $P_{k}$ 's. We first note that if all the $d_{i}$ 's in any of the 3 diagonal blocks $\left[\begin{array}{ccc}d_{r} & 0 & 0 \\ 0 & d_{s} & 0 \\ 0 & 0 & d_{t}\end{array}\right]$ are nonzero, then rank $A \geq 3$. So suppose each of these diagonal blocks has at least one $d_{i}$ equal to 0 . Then $A$ has a principal submatrix of the form

$$
\left[\begin{array}{lll}
0 & a & c \\
a & 0 & b \\
c & b & 0
\end{array}\right], \quad a, b, c \neq 0
$$

Since the determinant is $2 a b c$, this is invertible if char $F \neq 2$ and we conclude again that rank $A \geq 3$. Now consider the matrix

$$
B=\left[\begin{array}{lll}
0_{3} & J_{3} & J_{3} \\
J_{3} & 0_{3} & J_{3} \\
J_{3} & J_{3} & 0_{3}
\end{array}\right] \in S\left(F, K_{3,3,3}\right)
$$


Clearly, rank $B \leq 3$. If char $F \neq 2$,

$$
\operatorname{rank} B \geq \operatorname{rank}\left[\begin{array}{ccc}
0 & 1 & 1 \\
1 & 0 & 1 \\
1 & 1 & 0
\end{array}\right]=3
$$

while if $\operatorname{char} F=2$, rank $B=2$. We summarize the results of this section.

Observation 6 Let $F$ be a field.

(a) $\operatorname{mr}\left(F, P_{4}\right)=\operatorname{mr}(F, \ltimes)=\operatorname{mr}(F$, dart $)=\operatorname{mr}\left(F, P_{3} \cup K_{2}\right)=\operatorname{mr}\left(F, 3 K_{2}\right)=3$.

(b) $\operatorname{mr}\left(F, K_{3,3,3}\right)= \begin{cases}3 & \text { if char } F \neq 2, \\ 2 & \text { if char } F=2 .\end{cases}$

Consequently, if $G$ is a graph with $\operatorname{mr}(F, G) \leq 2$, none of the graphs $P_{4}, \ltimes$, dart, $P_{3} \cup$ $K_{2}, 3 K_{2}$ is an induced subgraph of $G$. We call such graphs $\left(P_{4}, \ltimes\right.$, dart, $\left.P_{3} \cup K_{2}, 3 K_{2}\right)$ free. If, in addition, char $F \neq 2, K_{3,3,3}$ is also not an induced subgraph of $G$. We will later see (Theorem 6 ) that this is a complete list of forbidden subgraphs for the class of minimum rank 2 graphs when char $F \neq 2$ and $F$ is infinite. We shall also see that if char $F=2$, then $K_{3,3,3}$ is replaced by $\left(P_{3} \cup 2 K_{3}\right)^{c}$.

3. Nondegenerate, bilinear symmetric forms on $\boldsymbol{F}^{\mathbf{2}}$. A line in $F^{2}$ is a one dimensional subspace of $F^{2}$. We let $S_{2}(F)$ denote the symmetric $2 \times 2$ matrices over $F$.

Let $B=\left[b_{i j}\right] \in S_{2}(F)$ be invertible. Then $B$ defines a nondegenerate, bilinear symmetric form

$$
(x, y) \mapsto x^{t} B y, \quad x, y \in F^{2} .
$$

Given any line $L$ in $F^{2}$ define its orthogonal complement (relative to the given form) by

$$
L^{\perp}=\left\{y \in F^{2} \mid y^{t} B x=0 \quad \forall x \in L\right\} .
$$

Since $B$ is invertible, $L^{\perp}$ is a line in $F^{2}$.

\section{Observation 7}

(i) $y \in L^{\perp} \Leftrightarrow y^{t} B u=0$ where $\{u\}$ is a basis of $L$.

(ii) $\left(L^{\perp}\right)^{\perp}=L$.

It is possible that $L^{\perp}=L$. In that case we say $L$ is an isotropic line.

Now we consider the existence and the number of isotropic lines. For this purpose we can replace $B \in S_{2}(F)$ by any matrix congruent to it. We distinguish two cases.

Case (I) $\operatorname{char} F \neq 2$.

Here we can assume $B$ is a diagonal matrix, and since we may replace $B$ by $\frac{1}{\alpha} B$ for any $\alpha \in F^{*}$, we can assume $B=\operatorname{diag}(1, d), d \in F^{*}$. A line $L=\operatorname{Span}\{x\}$ is isotropic if and only if

$$
x_{1}^{2}+d x_{2}^{2}=0
$$


Thus, there exist isotropic lines iff $-d=\beta^{2}$ for some $\beta \in F^{*}$. If this is the case, we have exactly two distinct isotropic lines, $S p\left\{\left[\begin{array}{l}x_{1} \\ x_{2}\end{array}\right]\right\}$ and $S p\left\{\left[\begin{array}{c}x_{1} \\ -x_{2}\end{array}\right]\right\}$, where $x_{1}, x_{2} \in$ $F^{*}$ and satisfy (3.1). Otherwise, there are no isotropic lines.

We note that for the case $F=\mathbb{R}$, isotropic lines exist if and only if $B$ has exactly one negative and one positive eigenvalue. So if $B$ is (positive or negative) definite, there are no isotropic lines.

Case (II) $\quad \operatorname{char} F=2$.

If $b_{11} \neq 0$ or $b_{22} \neq 0$, we can diagonalize $B$ by a congruence, and, as in the previous case, $L=\operatorname{span}\{x\}$ is isotropic if and only if equation (3.1) holds. If $-d$ is not a square, there is no isotropic line, and if $-d=\beta^{2}, x_{1}^{2}-\beta^{2} x_{2}^{2}=\left(x_{1}-\beta x_{2}\right)^{2}$, and there is one isotropic line, $x_{1}-\beta x_{2}=0$. If $b_{11}=b_{22}=0$, we can assume without loss of generality

$$
B=\left[\begin{array}{ll}
0 & 1 \\
1 & 0
\end{array}\right]
$$

so $x^{t} B y=x_{2} y_{1}+x_{1} y_{2}=x_{2} y_{1}-x_{1} y_{2}$. Hence every line $L$ is isotropic.

4. Graphs $G$ with $\operatorname{mr}(\boldsymbol{F}, G) \leq \mathbf{2}$. We need the following elementary result and include its proof for the sake of completeness.

Lemma 1 Let $A \in S_{n}(F)$ with rank two. Then there is an invertible $B \in S_{2}(F)$ such that $A=U^{t} B U$, where $U$ is a $2 \times n$ matrix.

Proof: We may assume without loss of generality that the first two columns of $A$ are linearly independent. Since $A$ has rank two, there is a $2 \times n$ matrix $W$ such that the matrix

$$
A\left[\begin{array}{cc}
I_{2} & W \\
0 & I_{n-2}
\end{array}\right]
$$

has columns $3,4, \ldots, n$ equal to 0 . Then

$$
\left[\begin{array}{cc}
I_{2} & W \\
0 & I_{n-2}
\end{array}\right]^{t} A\left[\begin{array}{cc}
I_{2} & W \\
0 & I_{n-2}
\end{array}\right]=\left[\begin{array}{cc}
B & 0 \\
0 & 0
\end{array}\right]
$$

with $B \in S_{2}(F)$ and invertible. Let $U=\left[\begin{array}{ll}I_{2} & -W\end{array}\right]$. Then $A=U^{t} B U$.

Theorem 1 Let $F$ be a field and $G$ a graph on $n$ vertices for which $\operatorname{mr}(F, G) \leq 2$.

1. If char $F \neq 2$, then $G^{c}$ is of the form

$$
\left(K_{s_{1}} \cup K_{s_{2}} \cup K_{p_{1}, q_{1}} \cup K_{p_{2}, q_{2}} \cup \cdots \cup K_{p_{k}, q_{k}}\right) \vee K_{r},
$$

for appropriate nonnegative integers $s_{1}, s_{2}, k, p_{1}, q_{1}, p_{2}, q_{2}, \ldots, p_{k}, q_{k}, r$ with $p_{i}+q_{i}>$ $0, i=1,2, \ldots, k$. 
2. If char $F=2$, then $G^{c}$ is either of the form

$$
\left(K_{s_{1}} \cup K_{s_{2}} \cup \cdots \cup K_{s_{k}}\right) \vee K_{r}
$$

or of the form

$$
\left(K_{s_{1}} \cup K_{p_{1}, q_{1}} \cup K_{p_{2}, q_{2}} \cup \cdots \cup K_{p_{k}, q_{k}}\right) \vee K_{r}
$$

for some appropriate nonnegative integers $k, s_{1}, s_{2}, \ldots, s_{k}, r, p_{1}, q_{1}, p_{2}, q_{2}, \ldots, p_{k}, q_{k}$ with $p_{i}+q_{i}>0, i=1,2, \ldots, k$.

Proof: The theorem is true if $\operatorname{mr}(F, G)=1$ by Observation 1 , so assume $\operatorname{mr}(F, G)=2$, and let $A \in S(F, G)$ with $\operatorname{rank} A=2$. By Lemma $1, A=U^{t} B U$, where $B$ is an invertible $2 \times 2$ symmetric matrix over $F$ and $U$ is $2 \times n$. For $i=1,2, \ldots, n$, let $w_{i}=\left[\begin{array}{l}x_{i} \\ y_{i}\end{array}\right]$ denote the $i$ th column of $U$ (so $\left[x_{i}, y_{i}\right]$ is the $i$ th row of $\left.U^{t}\right)$, and let

$$
L_{i}=S p\left\{w_{i}\right\}
$$

Consider now $1 \leq i \neq j \leq n$. We have

$$
i j \in E\left(G^{c}\right) \Leftrightarrow i j \notin E(G) \Leftrightarrow w_{i}^{t} B w_{j}=0 .
$$

Suppose that $r$ of the vectors $w_{1}, w_{2}, \ldots, w_{n}$ are 0 ; we may assume $w_{n-r+1}=w_{n-r+2}$ $=\cdots=w_{n}=0$. It follows that

$$
G^{c}=H \vee K_{r},
$$

where $H$ is the subgraph of $G^{c}$ induced by $\{1,2, \ldots, n-r\}$.

It remains to determine the structure of $H$. For $i=1,2, \ldots, n-r, L_{i}$ is a line, and it follows from (4.4) that for $j=1,2, \ldots, n-r$,

$$
i j \in E\left(G^{c}\right) \Leftrightarrow i j \notin E(G) \Leftrightarrow L_{j}=L_{i}^{\perp} \Leftrightarrow L_{i}=L_{j}^{\perp},
$$

where the orthogonal complement is with respect to the bilinear form defined by $B$.

1. Suppose that char $F \neq 2$. Then there are no isotropic lines or exactly two.

If there are two isotropic lines, denote them by $L^{(1)}$ and $L^{(2)}$. Consider now all $1 \leq i \leq n-r$ such that $L_{i}=L^{(1)}$. Suppose that there are $s_{1}$ such indices. Then $H$ must have a connected component which is $K_{s_{1}}$. Similarly, working with $L^{(2)}$ we conclude $\exists s_{2} \geq 0$ such that $K_{s_{2}}$ is another connected component of $H$.

Now pick any line among $L_{1}, L_{2}, \ldots, L_{n-r}$ which is not isotropic, say $L_{z}$, and consider all $1 \leq i \leq n-r$ such that $L_{i}=L_{z}$. Suppose we have $p_{1}$ such indices. We now consider all $1 \leq j \leq n-r$ such that $L_{j}=L_{i}^{\perp}$. Suppose we have $q_{1}$ such indices. Then $H$ must contain the component $K_{p_{1}, q_{1}}$. Repeating the process we complete the proof. (If there are no isotropic lines, $s_{1}=s_{2}=0$.)

2. Suppose that char $F=2$. Then there is at most one isotropic line or each line is isotropic. 
Let us first assume that each line is isotropic. Then $i j \in E(H)$ if and only if $L_{i}=L_{j}$. Hence $H$ is a disjoint union of complete graphs.

Assume now that there is at most one isotropic line, $L^{(1)}$. The vertices $i$ with $L_{i}=L^{(1)}$ induce a connected component which is a complete graph. The remainder of the proof is identical to the last paragraph in part 1.

Corollary 1 Let $G$ be a graph on $n$ vertices. Then if $\mathrm{mr}_{+}(G) \leq 2, G^{c}$ is of the form

$$
\left(K_{p_{1}, q_{1}} \cup K_{p_{2}, q_{2}} \cup \cdots \cup K_{p_{k}, q_{k}}\right) \vee K_{r}
$$

for appropriate nonnegative integers $k, p_{1}, q_{1}, p_{2}, q_{2}, \ldots, p_{k}, q_{k}, r$ with $p_{i}+q_{i}>0, i=$ $1,2, \ldots, k$.

Proof: The $B$ in the proof of Theorem 1 is now positive definite, so there are no isotropic lines. Then the components $K_{s_{1}}$ and $K_{s_{2}}$ in (4.1) are absent.

Theorem 2 Let $F$ be an infinite field.

1. If $\operatorname{char} F \neq 2$, let $G$ be any graph whose complement is of the form (4.1).

2. If $\operatorname{char} F=2$, let $G$ be any graph whose complement is of the form (4.2) or $(4.3)$.

Then $\operatorname{mr}(F, G) \leq 2$.

Proof: First, assume that char $F \neq 2$ and let $G^{c}$ have the form (4.1). Let $E=\left[\begin{array}{ll}0 & 1 \\ 1 & 0\end{array}\right]$. It suffices to show there exists a $U \in F^{2, n}$ such that $A=U^{t} E U \in S(F, G)$. We note that the symmetric, bilinear form corresponding to $E$ is given by

$$
\left[x_{1}, x_{2}\right] E\left[\begin{array}{l}
y_{1} \\
y_{2}
\end{array}\right]=x_{1} y_{2}+x_{2} y_{1} .
$$

Thus, the isotropic lines are $S p\left\{e_{1}=\left[\begin{array}{l}1 \\ 0\end{array}\right]\right\}$ and $S p\left\{e_{2}=\left[\begin{array}{l}0 \\ 1\end{array}\right]\right\}$.

Let $w_{i}, i=1,2, \ldots, n$ denote the columns of $U$. Choose $w_{n-r+1}=w_{n-r+2}=$ $\cdots=w_{n}=0$. Now let $w_{1}=w_{2}=\cdots=w_{s_{1}}=e_{1}$ and $w_{s_{1}+1}=w_{s_{1}+2}=\cdots=$ $w_{s_{1}+s_{2}}=e_{2}$.

Now pick $a \in F^{*}$ and let $x^{(1)}=\left[\begin{array}{l}1 \\ a\end{array}\right]$. Then, for $L=S p\left\{x^{(1)}\right\}$ we clearly have $L^{\perp}=S p\left\{y^{(1)}\right\}$, where $y^{(1)}=\left[\begin{array}{c}1 \\ -a\end{array}\right]$. So among the remaining $w_{i}$ 's we pick $p_{1}$ to be equal to $x^{(1)}$ and $q_{1}$ to be equal to $y^{(1)}$. We now choose $b \in F^{*}, b \neq a, b \neq-a$ and pick $p_{2}$ (resp. $q_{2}$ ) of the remaining $w_{i}$ 's to be equal to $\left[\begin{array}{l}1 \\ b\end{array}\right]\left(\operatorname{resp}\left[\begin{array}{c}1 \\ -b\end{array}\right]\right)$. Since $F$ is infinite and since the only zeros in the submatrix $A[\{1,2, \ldots, n-r\}]$ result when $x, y$ belong to the same isotropic line or belong to one of the pairs $L, L^{\perp}$ above, we can continue the process and obtain a matrix $A$ of rank $\leq 2$ in $S(F, G)$. 
We now assume that $\operatorname{char} F=2$. Consider first the case where the complement of $G$ is of the form

$$
\left(K_{s_{1}} \cup K_{s_{2}} \cup \cdots \cup K_{s_{k}}\right) \vee K_{r}
$$

Let $E=\left[\begin{array}{ll}0 & 1 \\ 1 & 0\end{array}\right]$. It suffices to show there exists a $U \in F^{2, n}$ such that $A=U^{t} E U \in$ $S(F, G)$. Each line in $F^{2}$ is isotropic. Let $w_{i}, i=1,2, \ldots, n$ denote the columns of $U$. Choose $w_{n-r+1}=w_{n-r+2}=\cdots=w_{n}=0$. Choose $k$ distinct lines $L_{1}, L_{2}, \ldots, L_{k}$ in $F^{2}$ and choose $v_{1}, v_{2}, \ldots, v_{k}$ such that $L_{i}=S p\left\{v_{i}\right\}, i=1,2, \ldots, k$. Let $w_{1}=w_{2}=$ $\cdots=w_{s_{1}}=v_{1}, w_{s_{1}+1}=w_{s_{1}+2}=\cdots=w_{s_{1}+s_{2}}=v_{2}, \ldots, w_{n-r-s_{k}+1}=\cdots=w_{n-r}=$ $v_{k}$. Then $U^{t} E U \in S(F, G)$.

We now assume that the complement of $G$ is of the form

$$
\left(K_{s_{1}} \cup K_{p_{1}, q_{1}} \cup K_{p_{2}, q_{2}} \cup \cdots \cup K_{p_{k}, q_{k}}\right) \vee K_{r} .
$$

Let $E=\left[\begin{array}{ll}1 & 0 \\ 0 & 1\end{array}\right]$. Notice that $S p\left\{e:=[1,1]^{t}\right\}$ is an isotropic line. Choose $w_{n-r+1}=$ $w_{n-r+2}=\cdots=w_{n}=0$. Choose $w_{1}, w_{2}, \ldots, w_{s_{1}}=e$.

Now pick $a \in F \backslash\{1\}$ and let $x^{(1)}=[1, a]^{t}$ and $y^{(1)}=[a, 1]^{t}$. Then $S p\left\{x^{(1)}\right\}$ is orthogonal to $S p\left\{y^{(1)}\right\}$. Among $w_{s_{1}+1}, w_{s_{1}+2}, \ldots, w_{n-r}$ we pick the first $p_{1}$ equal to $x^{(1)}$ and the $q_{1}$ after these equal to $y^{(1)}$. Since $F$ has an infinite number of elements, we can continue this process and obtain a matrix $A$ of rank $\leq 2$ in $S(F, G)$.

Theorem 2 is false if $F$ is finite.

Example Let $F$ be a finite field with $q$ elements and let $G^{c}=(q+2) K_{2}$. Suppose $\operatorname{mr}(F, G)=2$ (it can't be 1 ), and let $A \in S(F, G)$ with $\operatorname{rank} A=2$. By Lemma 1, $A$ can be factored $A=U^{t} B U$. Write $U=\left[w_{1}, w_{2}, \ldots, w_{2 q+4}\right] \in F^{2,2 q+4}$. If $0,1, a_{1}, a_{2}, \ldots, a_{q-2}$ are the elements of $F$, there are $q+1$ distinct lines in $F^{2}$, those spanned by $\left[\begin{array}{l}0 \\ 1\end{array}\right],\left[\begin{array}{l}1 \\ 0\end{array}\right],\left[\begin{array}{l}1 \\ 1\end{array}\right],\left[\begin{array}{c}1 \\ a_{1}\end{array}\right], \ldots,\left[\begin{array}{c}1 \\ a_{q-2}\end{array}\right]$. Consequently, there is a vertex $i$ in one $K_{2}$ and a vertex $j$ in another $K_{2}$ such that $w_{j}=\alpha w_{i}, \alpha \in F^{*}$. Since $i j$ is an edge of $G, w_{i}$ is not orthogonal to $w_{j}$, so it follows that $w_{i}$ is not isotropic. Let $k$ be the neighbor of $i$ in $G^{c}$. Then $w_{k} \perp w_{i}$, so $w_{k} \perp w_{j}$ which implies that $j k$ is an edge in $G^{c}$, a contradiction. Therefore $\operatorname{mr}(F, G) \geq 3$.

For the case $F=\mathbb{R}$ we have

Corollary 2 If $G$ is a graph whose complement is of the form (4.1), then $\operatorname{mr}(G) \leq 2$. Furthermore, if $\operatorname{mr}(G)=2$, the minimum is attained at a matrix with one positive and one negative eigenvalue.

Proof: This follows from Theorem 2, Theorem 1, and Sylvester's law of inertia.

Theorem 3 Let $G$ be a graph on $n$ vertices. Then $\mathrm{mr}_{+}(G) \leq 2$ if and only if $G^{c}$ has the form (4.5). 
Proof: The forward implication is Corollary 1. For the reverse implication, instead of adapting the proof of Theorem 2, we give a geometrical argument. Let $G$ be a graph of the form (4.5). Define a $2 \times n$ matrix $U=\left[u_{1}, u_{2}, \ldots, u_{n}\right]$ as follows. For each $i \in V\left(K_{r}\right)$, let $u_{i}=0$. Choose nonzero vectors $w_{1}, w_{2}, \ldots, w_{k} \in \mathbb{R}^{2}$ such that for $i \neq j, w_{i}$ and $w_{j}$ are linearly independent and $w_{i}^{t} w_{j} \neq 0$. Let $x_{1}, x_{2}, \ldots, x_{k}$ be nonzero vectors such that $w_{i}^{t} x_{i}=0, i=1,2, \ldots, k$. Let $S_{i}, T_{i}$ be the color classes of $K_{p_{i}, q_{i}}, i=1,2, \ldots, k$. For $v \in S_{i}$, define $u_{v}=w_{i}$ and for $v \in T_{i}$ define $u_{v}=x_{i}$. Then $U^{t} U \in S_{+}(G)$ and has rank $\leq 2$.

5. Forbidden Subgraph Characterizations. We now show how the graphs in (4.1), those in (4.2) and (4.3), and those in (4.5) can be characterized in terms of forbidden subgraphs through a series of propositions. It is clear that a graph $G$ is a union of complete graphs if and only if $G$ is $P_{3}$-free. Taking complements, a graph $G$ is a complete multipartite graph if and only if $G$ is $\left(K_{2} \cup K_{1}\right)$-free. It follows that $G$ is a complete bipartite graph if and only if $G$ is (triangle, $K_{2} \cup K_{1}$ )-free.

Proposition 1 The graph $G$ is a union of complete bipartite graphs if and only if $G$ is (triangle, $P_{4}$ )-free.

Forward implication. Since $G$ is bipartite, $G$ is triangle-free. If $P_{4}$ is an induced subgraph of $G$, it is an induced subgraph of some component, and then so is $K_{2} \cup K_{1}$, a contradiction.

Reverse implication. Let $H=(V, E)$ be a component of a (triangle, $P_{4}$ )-free graph $G$. Since $H$ is $P_{4}$-free, $H$ has no odd cycles, and is therefore bipartite. If $H$ is not complete bipartite, $P_{4}$ is an induced subgraph, contrary to assumption.

Proposition 2 Let $G=(V, E)$ be a connected paw-free graph containing a triangle. Then

1. every vertex of $G$ lies on a triangle.

2. $G$ does not contain a cut vertex.

Proof: Suppose $u$ is not on a triangle, let $k$ be the minimum distance from $u$ to a triangle, $G[\{w, x, y\}]$, and let $P=\left[u_{0}=u, u_{1}, u_{2}, \ldots, u_{k}=w\right]$ be a path from $u$ to the triangle. Since $G\left[\left\{u_{k-1}, w, x, y\right\}\right]$ is not a paw, we may assume $u_{k-1} x \in E$. Then $\left\{u_{k-1}, w, x\right\}$ induces a triangle at distance $k-1$ from $u$, a contradiction.

Now suppose $w$ is a cut vertex of $G$, and assume $\{w, x, y\}$ induces a triangle. Then there is a vertex $u$ adjacent to $w$ and lying in a component of $G-w$ distinct from the component containing $\{x, y\}$. Then $\{u, w, x, y\}$ induces a paw, contrary to assumption.

Proposition 3 A connected (paw, diamond)-free graph that contains a triangle is complete.

Proof: Let $G$ be such a graph. The proof is by induction on $n$, the number of vertices of $G$. If $n=3, G$ is $K_{3}$. Suppose $n \geq 4$ and that the theorem is true for all graphs with fewer than $n$ vertices. Assume $\left\{u_{1}, u_{2}, u_{3}\right\}$ induces a triangle and let $u$ 
be a vertex of $G$ distinct from $u_{1}, u_{2}, u_{3}$. By Proposition 2, $G-u$ is connected, so is complete by the induction hypothesis.

Since $G$ is connected, $u$ is adjacent to a vertex $x$. Let $y, z$ be any other vertices of $G-u$. Since $G-u$ is complete, $\{x, y, z\}$ induces a triangle. Since $G[\{u, x, y, z\}]$ is neither a paw nor a diamond, it is $K_{4}$, and $u$ is adjacent to $y$ and $z$. Consequently, $G$ is complete.

Proposition 4 A graph $G$ can be expressed as the union of complete graphs and of complete bipartite graphs if and only if $G$ is $\left(P_{4}\right.$, paw, diamond)-free.

Remark: The decomposition of a graph in this proposition is not unique. For example, $G=K_{4} \cup K_{3} \cup K_{2} \cup K_{2} \cup K_{2,2} \cup K_{1,5}$ can be thought of as the union of 4 complete graphs and 2 complete bipartite graphs, as the union of 2 complete graphs and 4 complete bipartite graphs, or of 3 of each.

Forward implication. None of $P_{4}$, paw, and diamond can be an induced subgraph of a component of $G$.

Reverse implication. We may express

$$
G=E_{1} \cup \cdots \cup E_{j} \cup H_{1} \cup \cdots \cup H_{k}
$$

where the $E_{i}$ and $H_{i}$ are the components of $G$, each $E_{i}$ contains a triangle, and each $H_{i}$ is (triangle, $P_{4}$ )-free. By Proposition 1 , each $H_{i}$ is a complete bipartite graph, and by Proposition 3 , each $E_{i}$ is complete.

Our next step is to characterize the class of graphs obtained by taking the join of a graph in Proposition 4 and a complete graph. Let $\hat{W}_{4}$ be the graph on 5 vertices

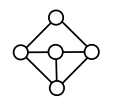

Theorem 4 A graph $G$ has the form

$$
\left(K_{s_{1}} \cup K_{s_{2}} \cup \cdots \cup K_{s_{t}} \cup K_{p_{1}, q_{1}} \cup K_{p_{2}, q_{2}} \cup \cdots \cup K_{p_{k}, q_{k}}\right) \vee K_{r}
$$

for nonnegative integers $t, s_{1}, s_{2}, \ldots s_{t}, k, p_{1}, q_{1}, p_{2}, q_{2}, \ldots, p_{k}, q_{k}, r$ with $p_{i}+q_{i}>0$, $i=1,2, \ldots, k$, if and only if $G$ is $\left(P_{4}\right.$, paw $\cup K_{1}$, diamond $\left.\cup K_{1}, \hat{W}_{4}, K_{2,2,2}\right)$-free.

Forward implication. Any induced subgraph of (5.1) containing a vertex of $K_{r}$ must contain a dominating vertex, so cannot be any of the graphs in

$$
\mathcal{F}=\left\{P_{4} \text {, paw } \cup K_{1} \text {, diamond } \cup K_{1}, \hat{W}_{4}, K_{2,2,2}\right\} .
$$

Therefore, if a graph in $\mathcal{F}$ is induced, it must be a subgraph of $K_{s_{1}} \cup K_{s_{2}} \cup \cdots \cup$ $K_{s_{t}} \cup K_{p_{1}, q_{1}} \cup K_{p_{2}, q_{2}} \cup \cdots \cup K_{p_{k}, q_{k}}$. Since the diamond is an induced subgraph of both $\hat{W}_{4}$ and $K_{2,2,2}$, it follows from Proposition 4 that no graph in $\mathcal{F}$ can be an induced 
subgraph of $K_{s_{1}} \cup K_{s_{2}} \cup \cdots \cup K_{s_{t}} \cup K_{p_{1}, q_{1}} \cup K_{p_{2}, q_{2}} \cup \cdots \cup K_{p_{k}, q_{k}}$. Therefore, a graph of the form (5.1) must be $\mathcal{F}$-free.

Reverse implication. Let $G=(V, E)$ be an $\mathcal{F}$-free graph. Let $D$ be the set of dominating vertices of $G$, let $r=|D|$, and let $C=V \backslash D$. Then $G=G[C] \vee K_{r}$. We now show that $G[C]$ is a (paw, diamond)-free graph.

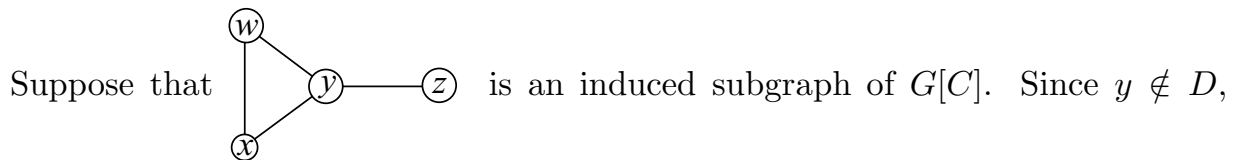

there exists a vertex $u \in C$ not adjacent to $y$. Since paw $\cup K_{1}$ is not induced, $u$ is adjacent to at least one of $w, x, z$. If $u$ is adjacent to a nonempty proper subset of $\{w, x, z\}, P_{4}$ is induced in $G[C]$, and therefore $G$, contrary to hypothesis, while if $u$ is adjacent to each of $w, x, z, \hat{W}_{4}$ is induced, also contrary to assumption. Therefore $G[C]$ is paw-free.

Suppose that<smiles>C1O[Co]1</smiles>
is an induced subgraph of $G[C]$. Since $x, y$ are not

dominating vertices, there exist vertices $a, b \in C$ with $a$ not adjacent to $y$ and $b$ not adjacent to $x$. If $a=b$, then since diamond $\cup K_{1}$ is not induced, $a$ is adjacent to at least one of $w$ and $z$, say $w$. Then $\{a, w, x, y\}$ induces a paw, a contradiction. So $a \neq b$. Then, since diamond $\cup K_{1}$ is not induced, $a$ is adjacent to at least one of $w, x, z$ and $b$ is adjacent to at least one of $w, y, z$. Suppose that $a$ and $x$ are not adjacent. Then, if $a$ is adjacent to only one of $w$ and $z, P_{4}$ is induced, and if $a$ is adjacent to $w$ and $z, \hat{W}_{4}$ is induced, both contrary to assumption. Therefore $a$ is adjacent to $x$, and by symmetry $b$ is adjacent to $y$. Then $a$ and $b$ are adjacent, else $\{a, x, y, b\}$ induces $P_{4}$. So

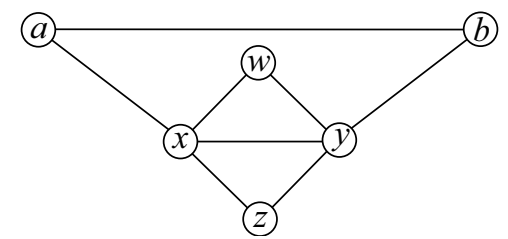

is a subgraph of $G[C]$. Since $G[C]$ is paw-free, $G[\{a, w, x, y\}]$ has more than 4 edges. But $a$ and $y$ are not adjacent, so $a w$ is an edge. A similar argument shows that $b w, a z$, and $b z$ are edges. Since $a y, b x, w z$ are not edges, $G[\{a, b, w, x, y, z\}]=K_{2,2,2}$, contrary to assumption. Therefore $G[C]$ is diamond-free.

Because $G[C]$ is (paw,diamond)-free and $P_{4}$-free by hypothesis, by Proposition 4 , $G[C]$ can be expressed as the union of complete graphs and complete bipartite graphs. Then $G=G[C] \vee K_{r}$ is of the form (5.1).

We can now give a forbidden subgraph characterization of the graphs in (4.1). 
Corollary 3 A graph $G$ has the form

$$
\left(K_{s_{1}} \cup K_{s_{2}} \cup K_{p_{1}, q_{1}} \cup K_{p_{2}, q_{2}} \cup \cdots \cup K_{p_{k}, q_{k}}\right) \vee K_{r}
$$

for nonnegative integers $s_{1}, s_{2}, k, p_{1}, q_{1}, p_{2}, q_{2}, \ldots, p_{k}, q_{k}, r$ with $p_{i}+q_{i}>0$, $i=1,2, \ldots, k$, if and only if $G$ is $\left(P_{4}\right.$, paw $\cup K_{1}$, diamond $\left.\cup K_{1}, \hat{W}_{4}, K_{2,2,2}, 3 K_{3}\right)$-free.

Note that the 6 forbidden graphs in this statement are the complements of the forbidden subgraphs in section 2 .

Forward implication. By Theorem 4 it suffices to show that $G$ is $3 K_{3}$-free. Since $3 K_{3}$ does not contain a dominating vertex, if it is an induced subgraph of $G$, it must be induced in $K_{s_{1}} \cup K_{s_{2}} \cup K_{p_{1}, q_{1}} \cup K_{p_{2}, q_{2}} \cup \cdots \cup K_{p_{k}, q_{k}}$. But $K_{3}$ is not an induced subgraph of a union of complete bipartite graphs, so $3 K_{3}$ must be induced in $K_{s_{1}} \cup K_{s_{2}}$, which is impossible.

Reverse implication. By Theorem 4, G has the form

$$
\left(K_{s_{1}} \cup K_{s_{2}} \cup \cdots \cup K_{s_{t}} \cup K_{p_{1}, q_{1}} \cup K_{p_{2}, q_{2}} \cup \cdots \cup K_{p_{k}, q_{k}}\right) \vee K_{r}
$$

We may assume that $s_{1}, s_{2}, \ldots, s_{t} \geq 3$. Then if $t \geq 3,3 K_{3}$ is induced. So $t \leq 2$ and $G$ has the form (5.2).

Taking complements in Corollary 3 we have

Theorem 5 Let $G$ be a graph. Then $G^{c}$ has the form (5.2) if and only if $G$ is $\left(P_{4}\right.$, dart, $\left.\ltimes, P_{3} \cup K_{2}, 3 K_{2}, K_{3,3,3}\right)$-free.

Combining Theorems $1,2(\operatorname{char} F \neq 2)$, and 5 ,

Theorem 6 Let $G$ be a graph and let $F$ be an infinite field such that char $F \neq 2$. Then the following are equivalent:

1. $\operatorname{mr}(F, G) \leq 2$.

2. $G^{c}$ has the form $\left(K_{s_{1}} \cup K_{s_{2}} \cup K_{p_{1}, q_{1}} \cup K_{p_{2}, q_{2}} \cup \cdots \cup K_{p_{k}, q_{k}}\right) \vee K_{r}$ for nonnegative integers $s_{1}, s_{2}, k, p_{1}, q_{1}, p_{2}, q_{2}, \ldots, p_{k}, q_{k}, r$ with $p_{i}+q_{i}>0, i=1,2, \ldots, k$.

3. $G$ is $\left(P_{4}, \ltimes\right.$, dart, $\left.P_{3} \cup K_{2}, 3 K_{2}, K_{3,3,3}\right)$-free.

In order to state analogous results when $\operatorname{char} F=2$ we need

Corollary 4 A graph $G$ has the form

$$
\left(K_{s_{1}} \cup K_{s_{2}} \cup \cdots \cup K_{s_{k}}\right) \vee K_{r}
$$

or has the form

$$
\left(K_{s_{1}} \cup K_{p_{1}, q_{1}} \cup K_{p_{2}, q_{2}} \cup \cdots \cup K_{p_{k}, q_{k}}\right) \vee K_{r}
$$

for some appropriate nonnegative integers $k, s_{1}, s_{2}, \ldots, s_{k}, r, p_{1}, q_{1}, p_{2}, q_{2}, \ldots, p_{k}, q_{k}$ with $p_{i}+q_{i}>0, i=1,2, \ldots, k$, if and only if $G$ is $\left(P_{4}\right.$, paw $\cup K_{1}$, diamond $\cup K_{1}$, $\left.\hat{W}_{4}, K_{2,2,2}, P_{3} \cup 2 K_{3}\right)$-free. 
Forward implication. It suffices by Theorem 4 to show that $P_{3} \cup 2 K_{3}$ is not induced. Suppose that it is. If $G$ is of the form (5.3), then since $P_{3} \cup 2 K_{3}$ does not have a dominating vertex, it must be induced in $K_{s_{1}} \cup K_{s_{2}} \cup \cdots \cup K_{s_{k}}$ implying that $P_{3}$ is induced in a complete graph, a contradiction. If $G$ is of the form (5.4), $P_{3} \cup 2 K_{3}$ must be induced in $K_{s_{1}} \cup K_{p_{1}, q_{1}} \cup K_{q_{2}, q_{2}} \cup \cdots \cup K_{p_{k}, q_{k}}$, implying that $2 K_{3}$ is induced in $K_{s_{1}}$, a second contradiction. Thus $P_{3} \cup 2 K_{3}$ is not induced.

Reverse implication. From Theorem 4 we see that $G$ has the form

$$
\left(K_{s_{1}} \cup K_{s_{2}} \cup \cdots \cup K_{s_{t}} \cup K_{p_{1}, q_{1}} \cup K_{p_{2}, q_{2}} \cup \cdots \cup K_{p_{k}, q_{k}}\right) \vee K_{r} .
$$

If $K_{s_{1}} \cup K_{s_{2}} \cup \cdots \cup K_{s_{t}} \cup K_{p_{1}, q_{1}} \cup K_{p_{2}, q_{2}} \cup \cdots \cup K_{p_{k}, q_{k}}$ is $P_{3}$-free, it is the union of complete graphs and $G$ has the form (5.3). So assume that $P_{3}$ is induced in $K_{p_{1}, q_{1}}$. Since $P_{3} \cup 2 K_{3}$ is not induced in $G, s_{i} \geq 3$ for at most one $i$. Then the remaining $K_{s_{i}}$ are complete bipartite graphs and $G$ has the form (5.4).

Combining Theorems 1, 2 ( $\operatorname{char} F=2$ ), and taking the complements of the graphs in Corollary 4 we have

Theorem 7 Let $G$ be a graph and let $F$ be an infinite field such that char $F=2$. Then the following are equivalent:

1. $\operatorname{mr}(F, G) \leq 2$.

2. $G^{c}$ is either of the form

$$
\left(K_{s_{1}} \cup K_{s_{2}} \cup \cdots \cup K_{s_{k}}\right) \vee K_{r}
$$

or of the form

$$
\left(K_{s_{1}} \cup K_{p_{1}, q_{1}} \cup K_{p_{2}, q_{2}} \cup \cdots \cup K_{p_{k}, q_{k}}\right) \vee K_{r}
$$

for some appropriate nonnegative integers $k, s_{1}, s_{2}, \ldots, s_{k}, r, p_{1}, q_{1}, p_{2}, q_{2}, \ldots$, $p_{k}, q_{k}$ with $p_{i}+q_{i}>0, i=1,2, \ldots, k$.

3. $G$ is $\left(P_{4}, \ltimes\right.$, dart, $\left.P_{3} \cup K_{2}, 3 K_{2},\left(P_{3} \cup 2 K_{3}\right)^{c}\right)$-free.

Theorems 6 and 7 are fairly definitive answers to the problem posed at the outset of the paper. The problem of characterizing which graphs have $\operatorname{mr}(F, G) \leq 2$ for finite fields is intricate and will be presented in a subsequent paper. The second criterion of these theorems is undoubtedly the characterization that should be used to algorithmically determine whether or not a graph has minimum rank less than or equal to 2 . The third criterion gives insight into the obstructions that prevent a graph from having rank less than 3 by establishing that the 6 forbidden graphs in section 2 comprise a complete list of minimal rank 3 graphs for an infinite field with $\operatorname{char} F \neq 2$ and the same list with one substitution is a complete list of minimal rank 3 graphs when char $F=2$. The equivalence of 2 and 3 in each theorem is of some interest graph theoretically as there is no transparent connection between them. Problems of finding forbidden subgraphs for graph classes obtained through unions and joins is investigated systematically in [1]. The problem of characterizing graphs with $\operatorname{mr}(F, G) \leq k$ for $k \geq 3$ appears very difficult. 
We can also give a forbidden subgraph characterization for the class of graphs $(4.5)$.

Corollary 5 A graph $G$ has the form

$$
\left(K_{p_{1}, q_{1}} \cup K_{p_{2}, q_{2}} \cup \cdots \cup K_{p_{k}, q_{k}}\right) \vee K_{r}
$$

for nonnegative integers $k, p_{1}, q_{1}, p_{2}, q_{2}, \ldots, p_{k}, q_{k}, r$ with $p_{i}+q_{i}>0, i=1,2, \ldots, k$, if and only if $G$ is $\left(P_{4}, K_{3} \cup K_{1}, \hat{W}_{4}, K_{2,2,2}\right)$-free.

Forward implication. Suppose $K_{3} \cup K_{1}$ were an induced subgraph. Since it has no dominating vertex, it would be induced in the union of complete bipartite graphs, which is impossible. Moreover, $P_{4}, \hat{W}_{4}$, and $K_{2,2,2}$ are all forbidden by Theorem 4 .

Reverse implication. Since $G$ is $\left(K_{3} \cup K_{1}\right)$-free, it is also (paw $\cup K_{1}$, diamond $\cup K_{1}$ )free. Then by Theorem $4, G$ has the form

$$
\left(K_{s_{1}} \cup K_{s_{2}} \cup \cdots \cup K_{s_{t}} \cup K_{p_{1}, q_{1}} \cup K_{p_{2}, q_{2}} \cup \cdots \cup K_{p_{k}, q_{k}}\right) \vee K_{r} .
$$

If $s_{1} \geq 3$ and $k \geq 1$, then $K_{3} \cup K_{1}$ is an induced subgraph, contrary to assumption. Suppose for some $i=1, \ldots, t$ that $s_{i} \geq 3$, so that $k=0$. If a second $s_{i}$ is positive, $K_{3} \cup K_{1}$ is again an induced subgraph. So all but one $s_{i}$ is 0 and $G$ is complete which is trivially of the form (5.7). So we may assume $s_{i} \leq 2$ for each $i$. But then all of the $K_{s_{i}}$ are bipartite and again $G$ has the form (5.7).

Combining Theorem 3 and Corollary 5 we have

Theorem 8 Given a graph $G$, the following are equivalent.

1. $\operatorname{mr}_{+}(G) \leq 2$.

2. $G^{c}$ has the form $\left(K_{p_{1}, q_{1}} \cup K_{p_{2}, q_{2}} \cup \cdots \cup K_{p_{k}, q_{k}}\right) \vee K_{r}$ for nonnegative integers $k, p_{1}, q_{1}, p_{2}, q_{2}, \ldots, p_{k}, q_{k}, r$ with $p_{i}+q_{i}>0, i=1,2, \ldots, k$.

3. $G$ is $\left(P_{4}\right.$, claw, $\left.P_{3} \cup K_{2}, 3 K_{2}\right)$-free.

We conclude this section by specializing our results to the case that $G$ is connected. Our first result is of some interest graph theoretically.

Proposition 5 Let $G$ be connected. Then $G$ is $\left(P_{4}\right.$, dart, $\left.\ltimes\right)$-free if and only if $G$ is $\left(P_{4}, P_{3} \cup K_{1}, K_{2} \cup 2 K_{1}\right)$-free.

Reverse implication. If the dart is an induced subgraph, so is $P_{3} \cup K_{1}$ and if $\ltimes$ is an induced subgraph, so is $K_{2} \cup 2 K_{1}$.

Forward implication. Suppose that $P_{3} \cup K_{1}=(x)(y)$ (2) (w) is an induced subgraph of $G$. Let $k$ be the minimum distance from $w$ to (x) (y)-(z). Then $k \geq 2$, but if $k>2, P_{4}$ is induced. Therefore $k=2$. Let $u$ be the intermediate vertex on a shortest path from $w$ to (x) (y) $z$, so that $u$ is adjacent to $w$ and at least one of $x, y, z$. If $u$ is adjacent to a proper subset of $\{x, y, z\}, P_{4}$ is induced, and if $u$ is adjacent to each of $x, y, z, G[\{x, y, z, u, w\}]$ is a dart. So $G$ is $\left(P_{3} \cup K_{1}\right)$-free. 
Suppose that $K_{2} \cup 2 K_{1}=(1)-(x)(1)(z)$ is induced. If the distance from $y$ to $\left(W-(X)\right.$ is greater than $2, P_{4}$ is induced, so the distance is 2 . Let $u$ be the intermediate vertex on a shortest path from $y$ to $\left(-X\right.$. If $u$ is not adjacent to both $w$ and $x, P_{4}$ is induced, so assume $u$ is adjacent to both. Then

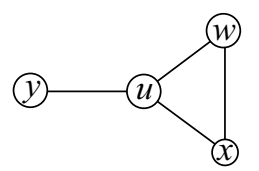

is an induced subgraph of $G$. If $z$ is adjacent to $u$, then $G[w, x, u, y, z]$ is $\ltimes$, a contradiction. So $z$ and $u$ are not adjacent. Replacing $y$ by $z$ in the argument with $y$ and $(1)-(x)$, there is a vertex $v \neq u$ such that

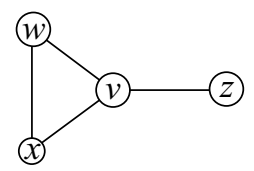

is an induced subgraph of $G$. If $u$ and $v$ are not adjacent, $G[u, w, v, z]$ is $P_{4}$, so $u$ and $v$ are adjacent. If $v$ and $y$ are not adjacent, $G[y, u, v, z]$ is $P_{4}$, so $v$ and $y$ are adjacent. Then $G[w, x, v, y, z]$ is $\ltimes$, a contradiction. So $K_{2} \cup 2 K_{1}$ is not induced.

If char $F \neq 2$, then from Theorem 6 we see that $G$ is connected only if $r=0$. We now characterize this class of graphs.

Proposition 6 A graph can be expressed as the union of at most two complete graphs and of complete bipartite graphs if and only if $G$ is $\left(P_{4}\right.$, paw, diamond, $\left.3 K_{3}\right)$-free.

Forward implication. $G$ is $\left(P_{4}\right.$, paw, diamond)-free by Proposition 4 , and $3 K_{3}$ is not induced since $K_{3}$ is not induced in a bipartite graph.

Reverse implication. By Proposition 4,

$$
G=K_{s_{1}} \cup K_{s_{2}} \cup \cdots \cup K_{s_{t}} \cup K_{p_{1}, q_{1}} \cup K_{p_{2}, q_{2}} \cup \cdots \cup K_{p_{k}, q_{k}},
$$

where we may assume $s_{1}, s_{2}, \ldots, s_{t} \geq 3$. Since $3 K_{3}$ is not induced, $t \leq 2$.

Taking complements we have

Corollary $6 G^{c}$ can be expressed as the union of at most two complete graphs and of complete bipartite graphs if and only if $G$ is $\left(P_{4}, P_{3} \cup K_{1}, K_{2} \cup 2 K_{1}, K_{3,3,3}\right)$-free.

We tighten this up by using the fact that $G$ is connected.

Corollary 7 Let $G$ be connected. Then $G$ is $\left(P_{4}\right.$, dart, $\left.\ltimes, K_{3,3,3}\right)$-free if and only if $G$ is $\left(P_{4}, P_{3} \cup K_{1}, K_{2} \cup 2 K_{1}, K_{3,3,3}\right)$-free.

Proof: This is an immediate consequence of Proposition 5.

Combining Corollaries 6 and 7, we have 
Corollary 8 If $G$ is a connected graph, then $G^{c}$ can be expressed as the union of at most two complete graphs and of complete bipartite graphs if and only if $G$ is $\left(P_{4}\right.$, dart, $\left.\ltimes, K_{3,3,3}\right)$-free.

Therefore, in the connected case, Theorem 6 becomes

Theorem 9 Let $G$ be a connected graph and let $F$ be an infinite field with char $F \neq 2$. Then the following are equivalent:

1. $\operatorname{mr}(F, G) \leq 2$.

2. $G^{c}$ can be expressed as the union of at most 2 complete graphs and of complete bipartite graphs.

3. $G$ is $\left(P_{4}\right.$, dart, $\left.\ltimes, K_{3,3,3}\right)$-free.

We can obtain analogues of Proposition 6 and Corollary 6 for the case char $F=2$.

Proposition 7 A graph can be expressed as the union of complete graphs or as the union of at most one complete graph and of complete bipartite graphs if and only if $G$ is $\left(P_{4}\right.$, paw, diamond, $\left.P_{3} \cup 2 K_{3}\right)$-free.

Forward implication. $G$ is $\left(P_{4}\right.$, paw, diamond)-free by Proposition 4 and it is easy to check that $P_{3} \cup 2 K_{3}$ is not induced.

Reverse implication. If $G$ is $P_{3}$-free, then $G$ is a union of complete graphs. So we may assume that $G$ has $P_{3}$ as an induced subgraph. By Proposition $4, G$ is the union of complete graphs and of complete bipartite graphs with $P_{3}$ induced in one of the complete bipartite graphs. Then $2 K_{3}$ cannot be induced, which means that at most one of the complete graphs has 3 vertices. Then $G$ can be expressed as the union of at most one complete graph and of complete bipartite graphs.

Taking complements we have

Corollary $9 G^{c}$ can be expressed as the union of complete graphs or as the union of at most one complete graph and of complete bipartite graphs if and only if $G$ is $\left(P_{4}, P_{3} \cup K_{1}, K_{2} \cup 2 K_{1},\left(P_{3} \cup 2 K_{3}\right)^{c}\right)$-free.

Proposition 8 Let $G$ be connected. Then $G$ is $\left(P_{4}\right.$, dart, $\left.\ltimes,\left(P_{3} \cup 2 K_{3}\right)^{c}\right)$-free if and only if $G$ is $\left(P_{4}, P_{3} \cup K_{1}, K_{2} \cup 2 K_{1},\left(P_{3} \cup 2 K_{3}\right)^{c}\right)$-free.

Proof: This follows immediately from Proposition 5.

Combining with Corollary 9, we have

Corollary 10 If $G$ is a connected graph, then $G^{c}$ can be expressed as the union of complete graphs or as the union of at most one complete graph and of complete bipartite graphs if and only if $G$ is $\left(P_{4}\right.$, dart, $\left.\ltimes,\left(P_{3} \cup 2 K_{3}\right)^{c}\right)$-free. 10

In the connected case we must have $r=0$ in Theorem 7. So we have by Corollary 
Theorem 10 Let $G$ be a connected graph and let $F$ be an infinite field with $\operatorname{char} F=$

2. Then the following are equivalent:

1. $\operatorname{mr}(F, G) \leq 2$.

2. $G^{c}$ can be expressed as the union of complete graphs or as the union of at most one complete graph and of complete bipartite graphs.

3. $G$ is $\left(P_{4}\right.$, dart, $\left.\ltimes,\left(P_{3} \cup 2 K_{3}\right)^{c}\right)$-free.

Finally, $G$ is connected in Theorem 8 only if $r=0$. Making use of Proposition 1, we have

Theorem 11 Given a connected graph $G$, the following are equivalent.

1. $\mathrm{mr}_{+}(G) \leq 2$.

2. $G^{c}$ is the union of complete bipartite graphs.

3. $G$ is $\left(K_{3}^{c}, P_{4}\right)$-free. $\left[G\right.$ is $P_{4}$-free with independence number $\leq 2$.]

6. Minimum Hermitian rank. In the previous sections we characterized for any infinite field $F$ those graphs $G$ such that $\operatorname{mr}(F, G) \leq 2$. This included the case $F=\mathbb{C}$. But besides symmetric matrices with entries in $\mathbb{C}$, we could also study the case of Hermitian matrices. Given a graph $G=(V, E)$ with vertex set $V=\{1,2, \ldots, n\}$, let $H(G)$ be the set of all Hermitian $n \times n$ matrices $A=\left[a_{i j}\right]$ such that $a_{i j} \neq 0, i \neq j$, if and only if $i j \in E$. There is no restriction on the diagonal entries of $A$. We define

$$
\operatorname{hmr}(G)=\min \{\operatorname{rank} A \mid A \in H(G)\} .
$$

Inspection of the six graphs presented in Section 2 shows that all of them except $K_{3,3,3}$ have $\operatorname{hmr}(G)=3$, and that $\operatorname{hmr}\left(K_{3,3,3}\right)=2$. In this section we characterize those graphs $G$ with $\operatorname{hmr}(G) \leq 2$. We will see that the forbidden subgraphs are the first five graphs presented in Section 2.

A line in $\mathbb{C}^{2}$ is a one dimensional subspace of $\mathbb{C}^{2}$ over $\mathbb{C}$. Let $B=\left[b_{i j}\right]$ be any invertible Hermitian $2 \times 2$ matrix. Given any line $L$ in $\mathbb{C}^{2}$ define its orthogonal complement (relative to $B$ ) by

$$
L^{\perp}=\left\{y \in \mathbb{C}^{2} \mid y^{*} B x=0 \quad \forall x \in L\right\} .
$$

Observation 7 (with $y^{t}$ replaced by $y^{*}$ ) is also valid for this case. We call a line $L$ isotropic if $L^{\perp}=L$.

We now consider the existence and the number of isotropic lines. For this we may replace $B$ by any matrix of the form $S^{*} B S$, where $S$ is a $2 \times 2$ invertible matrix over $\mathbb{C}$; we may also multiply it by -1 . Therefore, we can assume that either $B=\operatorname{diag}(1,-1)$ or $B=\operatorname{diag}(1,1)$. Let $\left[x_{1}, x_{2}\right]^{t}$ be a nonzero vector in $L$. Then $L$ is isotropic if and only if $x^{*} B x=0$. Thus, there exist isotropic lines if and only if $B=\operatorname{diag}(1,-1)$ and $\left|x_{1}\right|^{2}-\left|x_{2}\right|^{2}=0$. If this is the case, we can have $x_{1} \neq 0$ and $x_{2} \neq 0$; we may take $x_{1}=1$, and then $L$ is an isotropic line if and only if $\left|x_{2}\right|^{2}=1$. In particular, there can be infinitely many isotropic lines. 
Theorem 12 Let $G$ be a graph on $n$ vertices. Then, if $\operatorname{hmr}(G) \leq 2, G^{c}$ is of the form

$$
\left(K_{s_{1}} \cup K_{s_{2}} \cup \cdots \cup K_{s_{t}} \cup K_{p_{1}, q_{1}} \cup K_{p_{2}, q_{2}} \cup \cdots \cup K_{p_{k}, q_{k}}\right) \vee K_{r}
$$

for nonnegative integers $t, s_{1}, s_{2}, \ldots s_{t}, k, p_{1}, q_{1}, p_{2}, q_{2}, \ldots, p_{k}, q_{k}, r$ with $p_{i}+q_{i}>0$, $i=1,2, \ldots, k$.

The proof of this theorem is similar to the proof of Theorem 1, except that in this case there are an infinite number of isotropic lines if there are isotropic lines.

Theorem 13 Let $G$ be any graph whose complement is of the form (6.1). Then $\operatorname{hmr}(G) \leq 2$.

Proof: Let $E=\left[\begin{array}{cc}1 & 0 \\ 0 & -1\end{array}\right]$. It suffices to show there exists a $U \in \mathbb{C}^{2, n}$ such that $A=U^{*} E U \in H(G)$. Let $w_{i}, i=1,2, \ldots, n$ denote the columns of $U$, and let $e_{\zeta}=\left[\begin{array}{l}1 \\ \zeta\end{array}\right]$. If $\zeta \in \mathbb{C}$ and $|\zeta|=1$, the line spanned by $e_{\zeta}$ is isotropic. Choose $t$ distinct complex numbers $\zeta_{1}, \zeta_{2}, \ldots, \zeta_{t}$ with $\left|\zeta_{i}\right|=1$ for $i=1,2, \ldots, t$. Choose $w_{n-r+1}=w_{n-r+2}=$ $\cdots=w_{n}=0$. For $i=1,2, \ldots, t$, let $f_{i}=1+\sum_{j=1}^{i-1} s_{j}$ (with the understanding that $\left.f_{1}=1\right)$, let $l_{i}=\sum_{j=1}^{i} s_{j}$, and choose $w_{f_{i}}, w_{f_{i}+1}, \ldots, w_{l_{i}}=e_{\zeta_{i}}$.

Now pick a nonzero $\alpha_{1} \in \mathbb{C}$ with $\left|\alpha_{1}\right| \neq 1$. Then $x^{(1)}=\left[1, \alpha_{1}\right]^{t} \in \mathbb{C}^{2}$ is a nonisotropic vector and $y^{(1)}=\left[\overline{\alpha_{1}}, 1\right]^{t}$ is a vector in $\mathbb{C}^{2}$ orthogonal to $x^{(1)}$. Among the remaining $w_{i}$ 's we pick the first $p_{1}$ equal to $x^{(1)}$ and the $q_{1}$ after these equal to $y^{(1)}$. Since $\mathbb{C} \backslash\{\zeta|| \zeta \mid=1\}$ has an infinite number of elements, we can continue this process and obtain a matrix $A$ of rank $\leq 2$ in $H(G)$.

Corollary 11 If $\operatorname{hmr}(G)=2$, the minimum is attained at a Hermitian matrix with one positive and one negative eigenvalue.

Combining Theorems 12, 13 and taking complements in Theorem 4 gives

Theorem 14 Let $G$ be a graph. Then the following are equivalent:

1. $\operatorname{hmr}(G) \leq 2$.

2. $G^{c}$ has the form $\left(K_{s_{1}} \cup K_{s_{2}} \cup \cdots \cup K_{s_{t}} \cup K_{p_{1}, q_{1}} \cup K_{p_{2}, q_{2}} \cup \cdots \cup K_{p_{k}, q_{k}}\right) \vee K_{r}$ for nonnegative integers $t, s_{1}, s_{2}, \ldots, s_{t}, k, p_{1}, q_{1}, p_{2}, q_{2}, \ldots, p_{k}, q_{k}, r$ with $p_{i}+q_{i}>$ $0, i=1,2, \ldots, k$.

3. $G$ is $\left(P_{4}, \ltimes\right.$, dart, $\left.P_{3} \cup K_{2}, 3 K_{2}\right)$-free.

Let $H_{+}(G)$ be the set of positive semidefinite Hermitian matrices in $H(G)$ and let

$$
\operatorname{hmr}_{+}(G)=\min \left\{\operatorname{rank} A \mid A \in H_{+}(G)\right\}
$$

Theorem 15 Let $G$ be a graph. Then the following are equivalent: 
1. $\operatorname{hmr}_{+}(G) \leq 2$.

2. $G^{c}$ has the form $\left(K_{p_{1}, q_{1}} \cup K_{p_{2}, q_{2}} \cup \cdots \cup K_{p_{k}, q_{k}}\right) \vee K_{r}$ for nonnegative integers $k, p_{1}, q_{1}, p_{2}, q_{2}, \ldots, p_{k}, q_{k}, r$ with $p_{i}+q_{i}>0, i=1,2, \ldots, k$.

3. $G$ is $\left(P_{4}\right.$, claw, $\left.P_{3} \cup K_{2}, 3 K_{2}\right)$-free.

Proof: As in the proof of Corollary 1, there are no isotropic lines, so $K_{s_{1}}, K_{s_{2}}, \ldots, K_{s_{k}}$ in equation $(6.1)$ are absent. Thus $(1) \Longrightarrow(2)$. That $(2) \Longrightarrow(1)$ and $(2) \Longleftrightarrow(3)$ follows from Theorem 8 .

Recall the definition of $\tau(G)$ from the introduction. Theorem 15 says that $\tau(G) \geq$ $n-2$ if and only if $G^{c}$ has the form $\left(K_{p_{1}, q_{1}} \cup K_{p_{2}, q_{2}} \cup \cdots \cup K_{p_{k}, q_{k}}\right) \vee K_{r}$ for nonnegative integers $k, p_{1}, q_{1}, p_{2}, q_{2}, \ldots, p_{k}, q_{k}, r$ with $p_{i}+q_{i}>0, i=1,2, \ldots, k$.

We now specialize the result of this section to the case that $G$ is connected. As before, we see that $G$ is connected only if $r=0$. Taking complements in Proposition 4 , we obtain

Corollary $12 G^{c}$ can be expressed as the union of complete graphs and of complete bipartite graphs if and only if $G$ is $\left(P_{4}, P_{3} \cup K_{1}, K_{2} \cup 2 K_{1}\right)$-free.

Applying Proposition 5, Theorem 14 for a connected graph becomes

Theorem 16 Let $G$ be a connected graph. Then the following are equivalent:

1. $\operatorname{hmr}(G) \leq 2$.

2. $G^{c}$ can be expressed as the union of complete graphs and of complete bipartite graphs.

3. $G$ is $\left(P_{4}\right.$, dart, $\left.\ltimes\right)$-free.

This final result is quite intuitive. In contrast with Theorems 9 and 10, for a connected graph in the Hermitian case, the only forbidden subgraphs to having minimal rank $\leq 2$ are easily found.

Acknowledgment. We thank Charles R. Johnson for helpful early discussions on minimum rank problems for the class $S(G)$, and we thank Douglas West for the idea of using a symbol to denote the second graph in Section 2, as it has no common simple name.

\section{REFERENCES}

[1] M. Barrus. A Forbidden Subgraph Characterization Problem and a Minimal-Element subset of Universal Graph Classes. Master's Thesis, 2004, Brigham Young University.

[2] Y. Colin de Verdière. Sur un nouvel invariant des graphes et un critere de planarite. J. Combin. Theory, Ser. B, 50:11-21, 1990. [English translation: On a new graph invariant and a criterion for planarity, in: Graph Structure Theory (N. Robertson, P. Seymour, eds.), Contemporary Mathematics, American Mathematical Society, Providence, Rhode Island, 1993, pp. 137-147.]

[3] Y. Colin de Verdière. Multiplicities of eigenvalues and tree-width of graphs. J. Combin. Theory Ser. B, 74: 121-146, 1998.

[4] M. Fiedler. A characterization of tridiagonal matrices. Linear Algebra Appl., 2:191-197, 1969. 
[5] H. van der Holst. A short proof of the planarity characterization of Colin de Verdière. $J$. Combin. Theory Ser. B, 65:269-272, 1995.

[6] H. van der Holst. Graphs whose positive semi-definite matrices have nullity at most two. Linear Algebra Appl., 375:1-11, 2003.

[7] H. van der Holst, L. Lovász, and A. Schrijver. The Colin de Verdière graph parameter. Graph theory and combinatorial biology (Balatonlelle, 1996), 29-85, Bolyai Soc. Math. Stud., 7, János Bolyai Math. Soc., Budapest, 1999.

[8] C.R Johnson and A.L. Duarte. The maximum multiplicity of an eigenvalue in a matrix whose graph is a tree. Linear and Multilinear Algebra, 46:139-144, 1999.

[9] C.R. Johnson, A.L. Duarte, C.M. Saiago, B.D. Sutton, and A.J. Witt. On the relative position of multiple eigenvalues in the spectrum of an Hermitian matrix with a given graph. Linear Algebra Appl., 363:147-159, 2003.

[10] C.R. Johnson and C.M. Saiago. Estimation of the maximum multiplicity of an eigenvalue in terms of the vertex degrees of the graph of a matrix. Electron. J. Linear Algebra, 9:27-31, 2002 (electronic).

[11] A. Leal-Duarte and C.R. Johnson. On the minimum number of distinct eigenvalues for a symmetric matrix whose graph is a given tree. Math. Inequal. Appl., 5:175-180, 2002.

[12] L. Lovász and A. Schrijver. A Borsuk theorem for antipodal links and a spectral characterization of linklessly embeddable graphs. Proceedings AMS., 126:1275-1285, 1998.

[13] L.Lovász and A. Schrijver. On the null space of a Colin de Verdiere matrix. Annales de l'Institute Fourier, 49:1017-1026. 1999. 